\title{
Incorporación de la literatura en un programa lectura del idioma inglés para desarrollar la competencia de escritura: Instituto Blanca Adriana Ponce Ponce de Tegucigalpa
}

\author{
Incorporation of Literature in the \\ English Language Reading Program to \\ Develop Writing Competencies: \\ Blanca Adriana Ponce Ponce Institute \\ From Tegucigalpa
}

\author{
Clara Elizabeth Chávez Suazo \\ Docente del Departamento de Letras-Inglés-UPNFM \\ chavezelizabeth940@yahoo.com \\ Asesora de Tesis: PhD. J enny Margoth Zelaya Matamoros \\ Docente Departamento de Letras y Lenguas-UPNFM \\ Agradecimiento especial al Fondo de Apoyo a la Investigaciçon de la UPNFM \\ por el financiamiento otorgado
}

\section{Resumen}

Esta es una visión general acerca de como la literatura ha sido incorporada en un programa de la enseñanza del inglés como lengua extranjera. Además de esto a través de esta propuesta se mostró la aplicación de una nueva metodología usando la literatura como una herramienta para desarrollar la competencia de escritura en el idioma inglés. Esta metodología consistió en escribir comentarios 
en el diario del alumno y hacer lectura intensiva usando mapas conceptuales para desarrollar la escritura. También la enseñanza del inglés en un colegio público fue un aspecto que se tomó en cuenta porque este estudio fue hecho en un colegio de Tegucigalpa, Honduras. Además de esto este fue un estudio de Investigación acción por eso los instrumentos y los procesos se basaron en lo que investigación acción sugiere para hacer un estudio educacional. Tres instrumentos fueron aplicados en este estudio: un test de suficiencia (placement test), una entrevista y un cuestionario. El resultado del desarrollo de la competencia escrita fue evidente en las diferentes asignaciones escritas dadas a los estudiantes.

Palabras clave: métodos de enseñanza, diario del alumno, diagrama sinóptico, conexiones de la lectura y escritura y la investigación acción.

Abstract: This is a general view about how Literature has been incorporated in a teaching EFL school program. In addition to this, through this proposal was shown the application of a new methodology using Literature as a tool for improving writing competence. This methodology consisted of; writing comments in the journal and doing intensive reading using the story map for improving writing. As well, teaching English in public school was an aspect taken into account, because the study was done in a public school in Tegucigalpa, Honduras. In addition to this it was an Action Research study so that all the instruments, and research processes were based on what AR suggests to do in an educational study. Three instruments were applied in this study; a placement test, an interview and a questionnaire. As a result the students' improvement was seen in the different journal's writing assignments given to them.

Key words: teaching methods, learner journal, story map, literature, writing $\&$ reading connections, and action research. 
Incorporación de la literatura en un programa lectura del idioma inglés para

desarrollar la competencia de escritura: Instituto Blanca Adriana Ponce de Tegucigalpa

\section{Introducción}

La enseñanza de inglés como lengua extranjera ha sido un tema de investigación porque siempre se está investigando en el aula de clase como desarrollar en los estudiantes un buena performance de las competencias del idioma inglés. Este estudio se enfocó en como la literatura podría mejorar la competencia de la escritura. En Honduras hasta ahora no ha habido ningún estudio que se refiriera a este problema en el idioma inglés. Por esta razón, esta investigación es un punto de partida para hacer más estudios en los salones de clase acerca de la enseñanza del inglés como lengua extranjera. Los resultados de la investigación enfatizan cuan útil puede ser el uso de la literatura en la enseñanza del inglés.

De acuerdo con Muñoz (2003), la literatura ha sido usada en otros países como; Suecia, Alemania, Italia, Dinamarca y España entre otros. Los educadores e investigadores sugieren que la literatura puede ser incluida en la enseñanza en la clase de inglés por sus beneficios y ventajas. Asimismo el desarrollo de la escritura en la lengua extranjera tiene una estrecha relación con las actividades de lectura. Para lograr este objetivo fue necesario preparar un programa de lectura el cual incluyó tres historias cortas, tres poemas y tres refranes. Después de realizar los ejercicios de lectura los estudiantes escribieron comentarios en sus diarios (journals) para desarrollar la competencia de escritura. Además del programa de lectura, los estudiantes del décimo grado utilizaron un libro de texto y de trabajo en la clase de inglés los cuales también incluían ej ercicios de escritura y lectura. Al final del la investigación los estudiantes del grupo experimental mejoraron su competencia escrita considerablemente.

Además de esto, la mayoría de los comentarios que los estudiantes hicieron en la entrevista acerca del uso de la literatura en la clase de inglés fueron positivos. Acerca del uso de la literatura se puede mencionar que los hallazgos mencionados por la Dra. Muñoz (2003) después de haber aplicado la literatura para desarrollar las competencias lingüísticas en inglés en los salones de las escuelas públicas pudo observar la mejora en sus estudiantes: Por esa razón ella sugiere que la literatura puede ser una herramienta beneficiosa 
Clara Elizabeth Chávez Suazo

para la clase de inglés.

\section{Marco teórico de la investigación}

Este estudio se basó en la aplicación de la literatura trabajo hecho por la Dra. Muñoz(2003) en España. El propósito principal de este estudio incluyó el uso de textos literarios en el desarrollo de la enseñanza del inglés en el salón de clase para desarrollar las competencias lingüísticas globales del idioma inglés. Sin embargo en este estudio el objetivo más importante fue el desarrollo de la competencia escrita a través de lectura de varios tipos de textos literarios. La mayoría de los aulas de clase de la enseñanza del inglés como lengua extranjera(EFL) se enfocan en la enseñanza de la gramática pero no como el punto toral el cual es el desarrollo de las competencias lingüísticas del idioma inglés. Sumado a esto el desarrollo de la competencia de escritura en inglés es sinónimo de estatus, educación además del desarrollo de aquellas competencias cognitivas las cuales son necesarias para ser un experto en dicha competencia. Escribir no es solo escribir una palabra o frase esta competencia también incluye los aspectos socioculturales, afectivos y personales. También, Bello (1997) sugiere lo siguiente acerca de la competencia de escritura "una de las mayores faltas en el Currículo Tradicional pudo haber contribuido a la falta de atención dada a la competencia de escritura la cual es una avenida importante para desarrollar el pensamiento".

En otras palabras a través de la competencia de escritura los estudiantes puede mostrar que ha aprendido un idioma cuando pueden comunicar sus ideas y pensamientos porque un idioma es que palabras, frases o pedazos de escritura. Los estudiantes se dan cuenta de cuánto han progresado en el proceso de aprendizaje, asimismo pueden revisar y corregir sus propios errores durante este proceso. Otro aspecto que se puede mostrar en la producción escrita es que los estudiantes transfieren las estructuras de su lengua materna tanto como los recursos de traducción los cuales son utilizados por los estudiantes cuando empiezan en el proceso de la producción escrita. 
Incorporación de la literatura en un programa lectura del idioma inglés para

desarrollar la competencia de escritura: Instituto Blanca Adriana Ponce de Tegucigalpa

\section{Objetivos de la investigación}

El propósito del estudio es profundizar a través de la investigación si el uso de la literatura como herramienta de enseñanza puede desarrollar la competencia de escritura en los estudiantes de décimo grado de un colegio público en la clase de inglés como idioma extranjero. A continuación se presentan los objetivos específicos.

Este estudio trató de responder las siguientes preguntas:

1. ¿Cuál es el nivel léxico y gramatical de los estudiantes de décimo grado antes y después de exponerlos a la literatura en inglés?

2. ¿Cuál es el nivel de escritura de los estudiantes antes y después de exponerlos a la literatura en inglés?

3. Cuál es la actitud de los estudiantes hacia la literatura en inglés en el desarrollo de la competencia escrita?

4. ¿Es efectivo el programa de lectura elaborado y validado para mejorar la competencia escrita en el aula de clase de un colegio público?

\section{Metodología}

El diseño de esta investigación se basó en los parámetros de la Investigación Acción según Burns (2009); este estudio fue hecho en un aula de clase de un colegio público de Tegucigalpa, Honduras.

\section{Metología del programa de lectura}

Este programa fue parte de un estudio para mejorar la lectura pero mayormente la competencia de escritura en el Colegio Público" Instituto Blanca Adriana Ponce" en Tegucigalpa. Los participantes fueron estudiantes de décimo grado que el sistema educativo es I de bachillerato en Ciencias y letras. Los temas a enseñar se relacionaron con la edad de los estudiantes y sus intereses. A través de este programa los estudiantes fueron expuestos a la lectura de textos literarios auténticos en el idioma inglés. El programa se basó en el hecho de que los estudiantes ya tenían nociones básicas del idioma inglés como lengua extranjera porque ellos ya habían estudiado la clase de inglés desde séptimo grado hasta el noveno 
grado. La implementación del programa de lectura fue aplicada durante todo un año académico, el cual empezó en febrero de 2011 y terminó en noviembre del mismo año.

El Programa de lectura tenía tres unidades y estas unidades estaban conectadas al mismo tiempo con el texto de inglés "Everything 3a”" (unidades 1.3. y 3) y las actividades extracurriculares que el instituto planeo en el año escolar según el calendario dado por él. La oficina departamental del Distrito No.5. Asimismo, los estudiantes podían encontrar más apoyo lingüístico y ejercicios en cada unidad de los libros de texto según el nivel en el cual aparecía las cuatro habilidades del inglés. Este libro de texto proveía actividades enfocadas a escuchar, hablar, lectura y escritura.

Asimismo, este Programa de Lectura hizo énfasis especialmente en desarrollar la competencia de lectura. La competencia de lectura ofrece habilidades y estrategias como: skimming, scanning, guessing the meaning a través del contexto, mapas conceptuales, ejercicios de selección múltiple y verdadero y falso entre otros. Sumado a esto en el libro de textos aparecen la gramática y el vocabulario de una manera contextualizada la cual permite desarrollar las competencias cognitivas y lingüísticas en el idioma inglés.

Es más el libro de texto sugiriere algunas actividades de lectura y escritura a través de las cuales los estudiantes pueden seguir el ejemplo para escribir otro texto usando el mismo esquema de trabajo. El proceso de la adquisición y comprensión del idioma fue medido en las unidades a través de la evaluación de las actividades sugeridas por el manual del maestro. Además de esto, la evaluación formativa (assessment) fue presentada a través de las tres unidades del programa de lectura y escritura.

\section{Los Participantes}

Los estudiantes que participaron en este estudio fueron un total de 76 de décimo grado; grupo experimental con 20 alumnas y 14 alumnos; el grupo de control con 20 alumnas y 22 alumnos sus edades estaban comprendidas en un rango de 16 a 20 años de 
Incorporación de la literatura en un programa lectura del idioma inglés para

desarrollar la competencia de escritura: Instituto Blanca Adriana Ponce de Tegucigalpa

edad. Estos estudiantes del Instituto Blanca Adriana Ponce eran de un nivel social bajo-medio

La metodología pedagógica fue aplicada solo al grupo experimental durante todo el año académico. A continuación se muestran algunos ejemplos de producción escrita de los estudiantes antes de la aplicación del nuevo tratamiento pedagógico.

\section{Recolección de los datos}

Para poder hacer aseveraciones o afirmaciones acerca de la enseñanza y aprendizaje, la investigadora debe colectar de manera sistemática los datos relevantes acerca del tema de estudio. Esta recolección y análisis de datos frecuentemente ocurre durante el proyecto de investigación acción. Este puede ocurrir por ejemplo al inicio para determinar las condiciones de actuales de la enseñanza y aprendizaje en la clase de inglés, luego conocer los cambios ocurridos a mediados del tiempo establecido de la aplicación del programa de lectura así como las líneas de cambio que se deben aplicar según las limitantes que se hayan presentado.

Posteriormente analizar el producto obtenido al final del año académico al terminar de aplicar este tratamiento pedagógico.

A continuación se presenta un cuadro sinóptico de cómo se llevó a cabo este estudio.

Figure No. 1

Instrument Stages \& Purposes

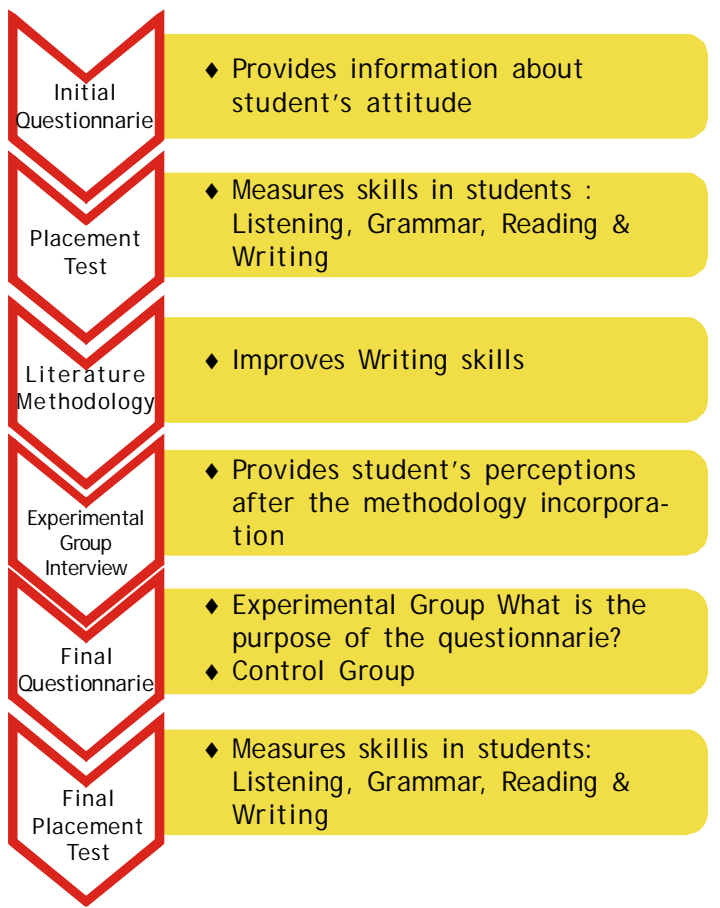


Clara Elizabeth Chávez Suazo

\section{Instrumentos}

Esta investigación requirió de tres instrumentos: un cuestionario inicial y final, una entrevista y un test de suficiencia de inglés (placement test) el cual se aplicó al inicio y al final del año académico. Estos instrumentos permitieron a la investigadora conocer primeramente sobre la actitud de los estudiantes hacia el idioma inglés aplicándosele a ambos grupos (experimental y de control). En segundo lugar se investigó cual era el nivel de inglés que los estudiantes tenían al inicio y al final de la aplicación de la nueva metodología. Finalmente, conocer cual era la percepción de los estudiantes acerca del uso de los textos literarios en la clase de inglés.

\section{Logros y limitaciones de la investigación}

En Honduras los paros laborales de los maestros son parte de los problemas en educaciones por eso que este factor fue considerado una limitante cuando se aplicaban test o cuando al gunas actividades eran hechas. Otro aspecto a considerar fue el número de los participantes y las ausencias en la clase. Este otro factor hizo imposible realizar algunas actividades porque aunque las actividades estuvieran planeadas y los materiales listos sucedían los paros laborales 0 alrededor de 5 a 8 alumnos no llegaban a clases ese día. Durante este año 2011 los paros laborales fueron bastantes largos. Esto hizo imposible que se cubrieran las cuatro unidades del Programa de Lectura y del texto de inglés.

Desafortunadamente, sólo dos unidades se cubrieron tanto del programa de lectura como del Libro de texto de inglés. Como consecuencias de los paros laborales en el año académico solamente se impartieron 40 horas de la clase de inglés. Otra limitante en la realización de las actividades planeadas con el Grupo Experimental el cual estaba en la jornada de la tarde fueron las condiciones del edificio porque las ventanas estaban quebradas y los chirridos de los carros no permitían escuchar los ejercicios de escucha en el aula de clase ya que esta estaba a la orilla del boulevard. Por otra parte el grupo de Control no tenia un aula disponible entonces tenían que recibir sus clases en el laboratorio de ciencias naturales el cual no era apropiado para recibir todas las clases por la mañana. 
Incorporación de la literatura en un programa lectura del idioma inglés para

desarrollar la competencia de escritura: Instituto Blanca Adriana Ponce de Tegucigalpa

Además de lo antes mencionado se debe mencionar el aspecto cultural ya que ambos grupos tenían estudiantes miskitos o garifunas. La mayor parte del tiempo los alumnos miskitos encontraban muy difíciles las actividades hechas en clases. No se debe obviar el hecho de para estos alumnos el inglés es una tercera lengua a aprender, esto por supuesto conlleva a buscar otras estrategias en el proceso de aprendizaje del inglés como idioma extranjero.

\section{Resultados y analisis de datos}

Este estudio duró todo el año académico del 2011. Después de la recolección se hizo el análisis de los datos. Durante el análisis de los datos se tomaron en cuenta los siguientes elementos de la investigación; el objetivo general, los objetivos específicos y las preguntas de investigación

Preguntas de Investigación

Esta investigación trata de responder las siguientes preguntas:

1) ¿Cuál es el nivel léxico y gramatical antes y después de ser expuestos a la literatura en ingles?

2) ¿Cual es el nivel de escritura en inglés antes y después de ser expuestos a la literatura en inglés?

3) ¿Cuál es la actitud del estudiante hacia la literatura en inglés en el desarrollo de la competencia escrita?

4) ¿Es efectivo el programa de lectura elaborado y validado para el desarrollo de la competencia escrita en inglés? 
Clara Elizabeth Chávez Suazo

Cuestionario Inicial, acerca del inglés como idioma extranjero: Actitud, Motivación y necesidades

\section{Gráfico No. 1}

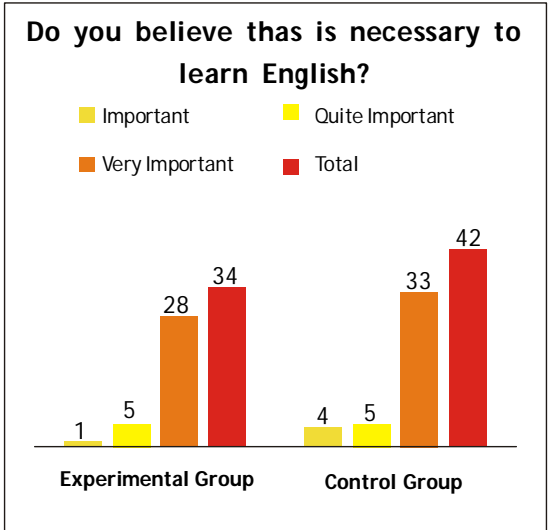

\section{Gráfico No.2}

What importance do yo give at English in relationship to the other subjects?

$$
\begin{aligned}
& \text { Nothing important } \quad \square \text { Important } \\
& \text { Quite important } \quad \square \text { Very important }
\end{aligned}
$$

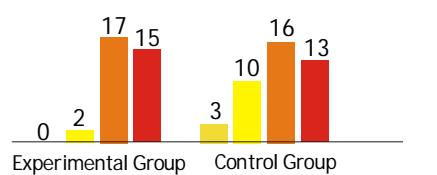

Experimental Group Control Group

\section{Gráfico No.3}

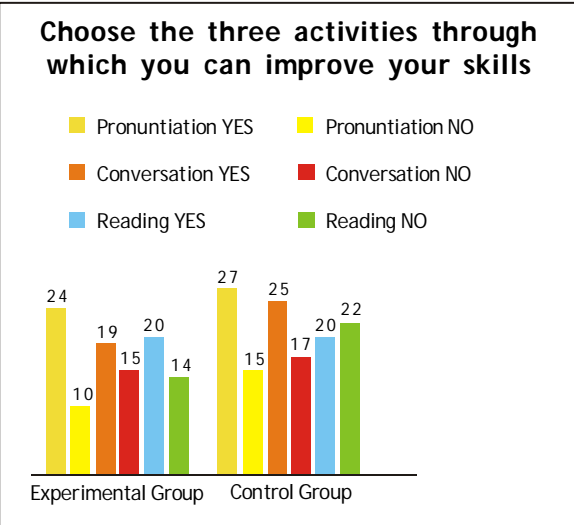

Análisis inicial de la performance en la competencia escrita

Producción de escritura Grupo Experimental

La respuesta de la segunda pregunta de investigación es:

¿Cuál es el nivel de escritura del estudiante antes y después de haber sido expuestos a la literatura en inglés? 
Incorporación de la literatura en un programa lectura del idioma inglés para

desarrollar la competencia de escritura: Instituto Blanca Adriana Ponce de Tegucigalpa

En el test inicial en la sección de composición los estudiantes escribieron un párrafo corto en el cual se muestra que las oraciones no fueron estructuradas gramaticalmente.

Por los estudiantes de ambos grupos. (Grupo experimental y de Control). Estos problemas se enfocaron en ortografía, gramática y puntuación. A continuación se muestran algunos ejemplos de la producción de los estudiantes.
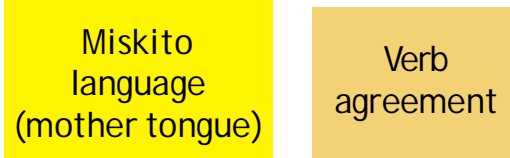

A. COMMUNICATION

SS44 "Your last name? Where do you live?

B. PUNCTUATION AND SPELLING

SS8 "teacher" "shildreen" instead of "children"; "inglis"istead of "English"

S552 "Where is the come Nou words"

SS56 "Helo Good morning ticher"

SS46 "school the school is the shildreen. my brother live teach ei to shildreen

SS74 "School liven you estudy is inglis

C. VOCABULARY

SS50 "Capital city Is Mall"

S557 "The school is Beautiful The school is big The school is red

SS59 "The school is big"

S566 "The classe ingles yes a my gustar aprender"

SS70 Miskito language: "You it's may laick is cul. May name is HA. You estudey en escull ceb Lempira you may laick escull dey ingles"

D. GRAMMAR

SS72 "I student in Blanca 
Clara Elizabeth Chávez Suazo

E. ORGANIZATION

SS64 "My favorit is and school is student My president is and capital city is bad"

F. TASK ACHIEVEMENT

SS48 "I m study in the house My name is ISP Am happy

Como se puede ver los estudiantes del grupo experimental muestra un competencia pobre en escritura. Sin embargo lo mejor de todo fue que su actitud hacia la lengua fue muy positiva como se muestra en los gráficos. Los estudiantes carecían de suficiente vocabulario y por eso no pudieron expresar una idea completa en sus escritos. Ellos tomaron palabras de su lengua materna. Mientras otros estudiantes los cuales pertenecían a un grupo étnico prefirieron escribir en su lengua materna como fue el caso de los estudiantes miskitos (SS 70)

El rendimiento de los estudiantes en escritura fue analizado de acuerdo a lo que estaba establecido en los ej emplos del "placement test" de inglés, el cual estaba basado en las siguientes categorías: comunicación. puntuación, ortografía, vocabulario, gramática, organización y logro en la tarea. Axbey (2005). Después de analizar los comentarios escritos por los estudiantes del grupo experimental en sus diarios y comparados luego con el párrafo final que hicieron cuando se les aplicó el test final se pudo observar que los estudiantes continuaron cometiendo errores en la producción escrita, pero el párrafo mostró ideas completas y coherentes.

\section{Resultados y discusión}

A continuación se muestran algunos de los productos de escritos al inicio del estudio. Se puede notar que los estudiantes de del grupo experimental tuvieron problemas con comunicación, vocabulario, puntuación, ortografía, gramática y organización entre otros. Además los estudiantes carecían de confianza en si mismos cuando escribían por primera vez bajo presión y en un idioma diferente al de ellos. Era evidente que los estudiantes no tenían suficiente vocabulario y entrenamiento acerca de cómo escribir un párrafo siguiendo las mecánicas requeridas en la lengua extranjera. Además 
Incorporación de la literatura en un programa lectura del idioma inglés para

desarrollar la competencia de escritura: Instituto Blanca Adriana Ponce de Tegucigalpa

de esto es importante resaltar cuan importante fue para los estudiantes del grupo experimental haber sido parte en la aplicación de esta nueva metodología y como ésta influyó en su actitud hacia el inglés como idioma extranjero y el uso de literatura en la clase de inglés. Los siguientes son algunos ej emplos de los comentarios hechos en la entrevista.

\section{Análisis de la entrevista}

Comentarios acerca del uso de la literatura en la clase de inglés y si consideraron el tema interesante:

a) En relación a este aspecto los estudiantes contestaron:

"Yes, topics seem very interesting, taking care of the animals, this theme was beautiful, and I liked it because the man took care of the bird (Gulliver), and he let it be free. The second one I liked too much because the old lady helped the kids in order to finish the match"

b) Los textos literarios mejoran la escritura: La percepción acerca de este aspecto fue claro porque ellos estuvieron consientes acerca de la mejora de la escritura en inglés.

"It helps us to improve the vocabulary and pronunciation"

"Yes, because each time that the teacher assigns a story we learn more"

"New words are discovered"

"We encourage writing because of the journal use, now I like very much English"

c) Textos literarios lo que les gusta y lo que no les gusta

"I liked the Three Musketeers because is a story that shows unity among them"

Cuestionario Final: Sección 1. Metodología y Evaluación de la Clase: este gráfico muestra la percepción de los estudiantes acerca del uso de los textos literarios. 
Gráfico No.4

To read and do the homework with the original short stories

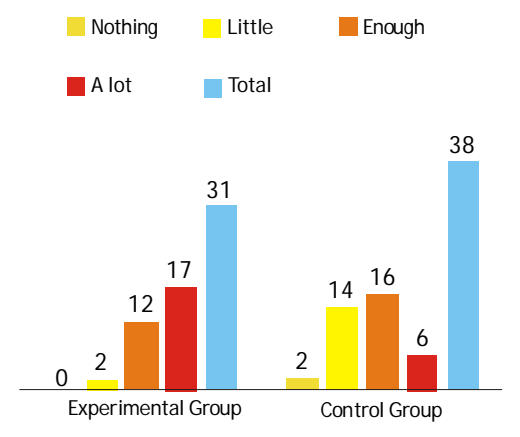

Gráfico No.5

H. It was intereting to write short paragraphs about the class topics, and literary texts?

Nothing Little Enough A lot

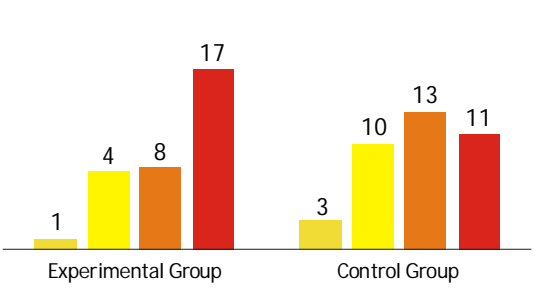

Finalmente, se pudo observar el rendimiento de los estudiantes al final de la incorporación de la nueva metodología hay un ejemplo de ambos grupos (experimental y de control). Hasta ahora los estudiantes no escribieron perfectamente bien porque cometieron los errores que comúnmente son hechos por los estudiantes cuando escriben en la lengua extranj era. (Transference problemas). Además de esto Pie de hierro(2009) menciona lo sugerido por Cummning acerca de este problema que para resolver el problema de comunicación los escritores desarrollan las competencias de escritura con las características de la L1 o lengua materna, luego estas características superficiales los estudiantes las aplican para escribir textos en la L2 o lengua meta. El dominio básico cognitivo 0 académico del nivel permite que los escritores transfieran las competencias de escritura de la lengua nativa a la lengua meta (p. 55) 
Incorporación de la literatura en un programa lectura del idioma inglés para

desarrollar la competencia de escritura: Instituto Blanca Adriana Ponce de Tegucigalpa

\section{Contrasting the final writing placement test between "Experimental Group \& Control Group"}

Figure No. 2
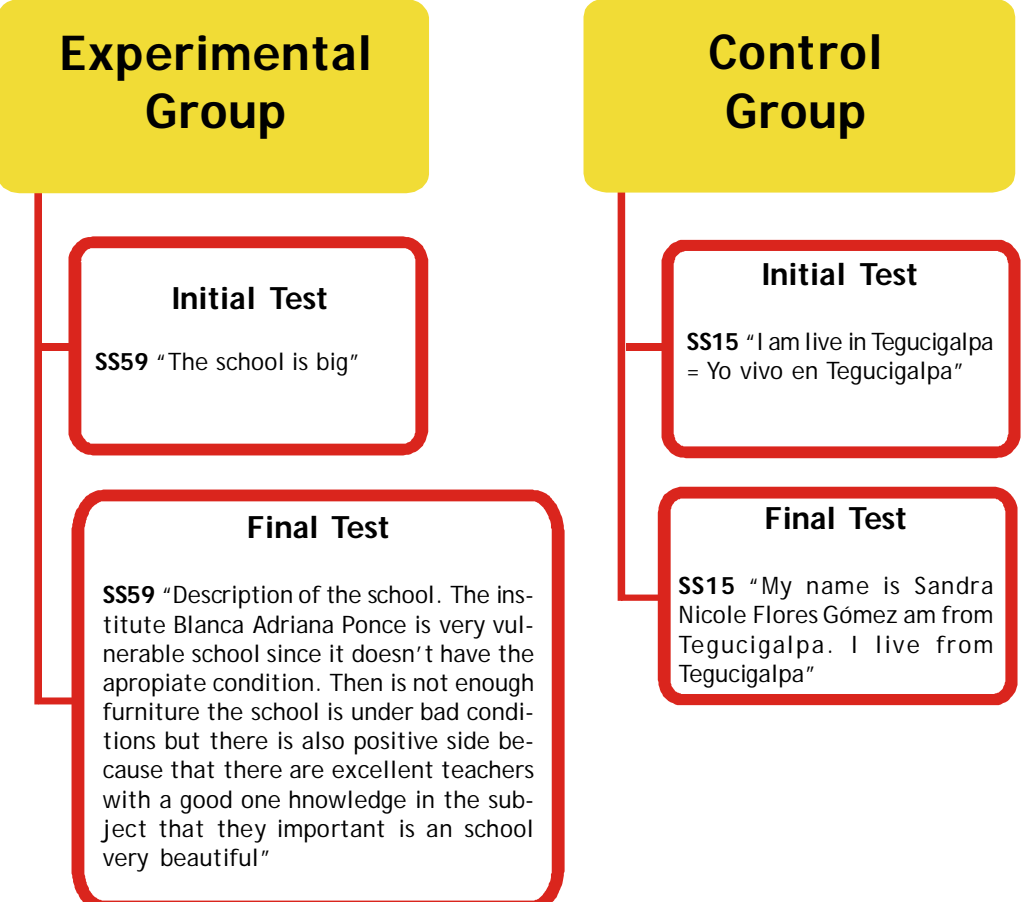
Figure No.3

\section{Experimental}

Group

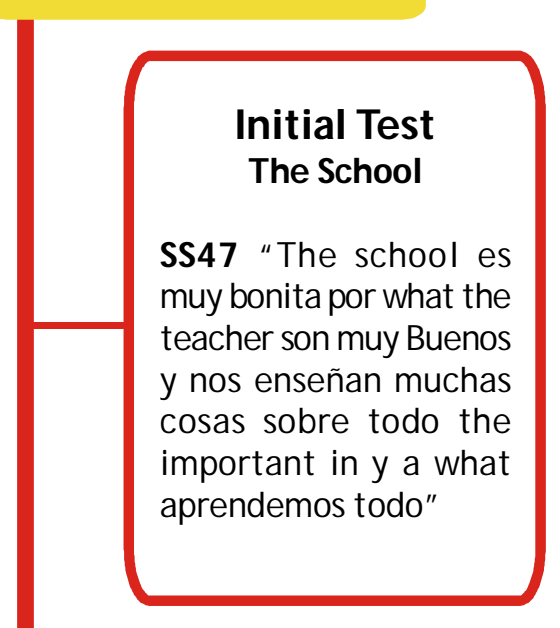

\section{Final Test \\ The School}

SS47 "My school is very pretty, my school you dut nat two laboratory one a laboratory of English a one laboratory of Science. My school is colors orange. My school is located kennedy. My school you dut not much teachers. I am my school takin of English on my teacher is Clara Luna. My school you dut hat library, cafetery. My school you dut hat career of Bachillerato Science y Letras, Hostelería y Turismo, Comercio. I am my school the class y English is very pretty ehat the teacher is very freaning. The class of English an like"

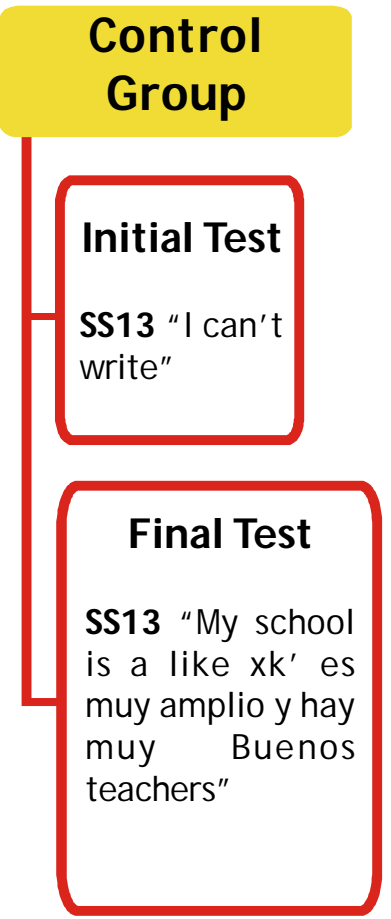

SS13 "My school is a like $x^{\prime}$ ' es muy amplio y hay muy Buenos teachers" 
Incorporación de la literatura en un programa lectura del idioma inglés para

desarrollar la competencia de escritura: Instituto Blanca Adriana Ponce de Tegucigalpa

\section{Conclusión}

Se concluye que la competencia de escritura fue desarrollada por los estudiantes con el uso de literatura en la clase de inglés. De acuerdo a Langer (1997), la literatura permite que los estudiantes reflexionen sobre sus vidas, aprendizaje e idioma. También la puntualiza en la relación que hay entre escritura y creatividad acerca de los cual Shorofar menciona lo sugerido por Tse y Shun (2000);"creatividad es un aspecto importante en la escritura". Tse y Sun puntualizan que la creatividad es considerada uno de los seis niveles de las habilidades lingüísticas. Asimismo, Lin(1998) dice que" La escritura es una forma de promover la creatividad del estudiante. Asimismo la creatividad y la generación de ideas es la forma más efectiva para desarrollar escritura"

También Shororfar (2007) clasifica la escritura como: -funcional y creativa" ? La escritura creativa es aquella en la cual los individuos expresan sus sentimientos, opiniones, reacciones e ideas para el lector en un distinguido estilo literario. Este tipo de textos incluye muchos géneros como; historias cortas, poemas, dramas, novelas, ensayos y descripciones-

El Placement Test mostró que los estudiantes tuvieron un pobre desarrollo en escucha, habla, lectura y escritura tanto en el grupo experimental como en el de control al inicio y al final de la investigación. En el grupo experimental la investigadora se enfocó en desarrollar escritura, lo opuesto al grupo de control al cual se le enseño sólo con el libro de texto de inglés y las actividades sugeridas por este. Por esta razón el test final mostró mejora en el grupo experimental pero no en el grupo de control.

En el grupo Experimental la actitud de los estudiantes hacia el uso de textos literarios en inglés fue aceptado por la mayoría de los estudiantes. No todos los estudiantes se sintieron cómodos con las lecturas de las historias cortas en inglés. Por esa razón clara y sinceramente ellos dij eron que no les gustaba leer ni en español ni inglés. Al inicio del estudio los estudiantes tuvieron miedo de leer los textos literarios porque ellos pensaban que sería muy difícil leer un artículo o historia corta. Finalmente cuando se les enseñó a ellos 
como leer una historia corta o un poema en inglés los estudiantes disfrutaron haciendo este tipo de actividades en el aula de clase 0 en casa.

También a los estudiantes se les permitió el uso del diccionario para traducir las palabras desconocidas para comprender de que se trataba la historia, o para hacer los ejercicios al final de cada actividad de lectura. Por último el Programa de Lectura fue elaborado y validado durante este estudio en el cual se aplicó la investigación acción. Este programa fue elaborado tomando en cuenta tanto los contenidos del libro de texto en inglés como las diferentes actividades extracurriculares realizadas durante el año académico. Los textos literarios trabajados en ingles fueron variados: poemas, refranes e historias cortas. Se cierra este apartado con lo citado por Clandfield (2009) ya que hay muchas razones para usar literatura en la clase de inglés entre ellas está el hecho de que los textos literarios frecuentemente ofrecen una rica gama de recursos de aprendizaje que pueden ser efectivos para promover discusiones, compartir sentimientos u opiniones entre los aprendices.

\section{Recomendaciones}

Después de analizar los resultados obtenidos de la aplicación del test la investigadora sugiere lo siguiente:

- El nivel léxico y gramatical de los estudiantes fue medido con el Placement Test el cual colocó a los estudiantes debajo del nivel de desempeño. Se recomienda que se unifiquen los contenidos de la clase de inglés según el Currículo Nacional Básico (CNB) en la clase de inglés en las tres jornadas del instituto.

- Se sugiere que las autoridades del instituto apoyen a los profesores de inglés para que se implemente este programa de lectura para desarrollar escritura en todos los cursos empezando con un grupo piloto. Porque cuando fue medida la competencia de escritura al inicio del estudio los estudiantes no pudieron escribir oraciones sencillas y sus ideas no eran claras acerca de los temas asignados. En el placement test los temas se relacionaban con la vida del estudiante (escuela, amigos, vecindario) sin embargo los resul tados obtenidos estuvieron por debajo de la nota sugerida 
Incorporación de la literatura en un programa lectura del idioma inglés para

desarrollar la competencia de escritura: Instituto Blanca Adriana Ponce de Tegucigalpa

por las escalas del test de inglés. Sin embargo al final del año académico se aplicó nuevamente el test a ambos grupos (experimental y de Control) y solamente el grupo experimental mostró una mej ora visible en la competencia de escritura porque el libro de texto fue trabajado de forma concatenada con el Programa de Lectura el cual se enfocó a desarrollar la competencia de escritura ayudada de las actividades de lectura.

- Este programa ayudó a los estudiantes a mejorar la competencia de escritura el cual puede ser aplicado en lectura, escucha y habla en la clase de inglés. También es importante destacar que los estudiantes se sintieron seguros cuando trabaj aron con textos literarios originales porque su actitud hacia la lengua fue muy positiva. El presente estudio mostró que si los estudiantes son enseñados en la forma apropiada con recursos literarios, ellos pudieran lograr los estándares propuestos en el Currículo Nacional Básico. 


\section{Anexos}

\section{WRITING TEST}

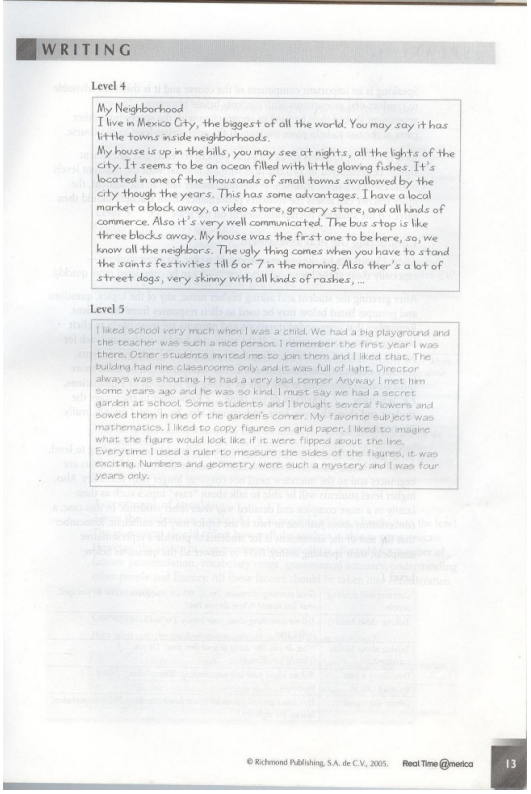

Ilustración 1

READING EXERCISE

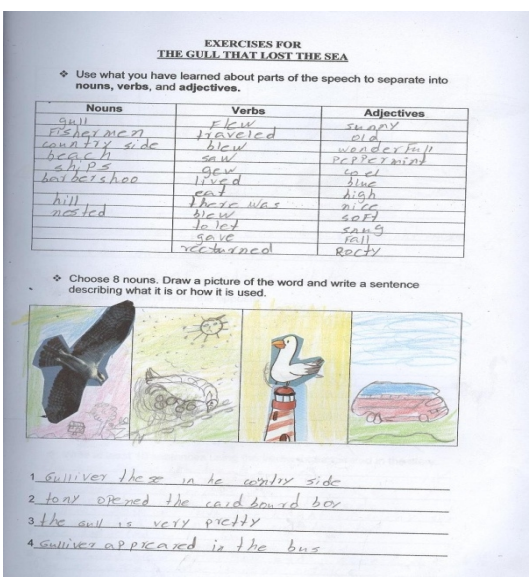

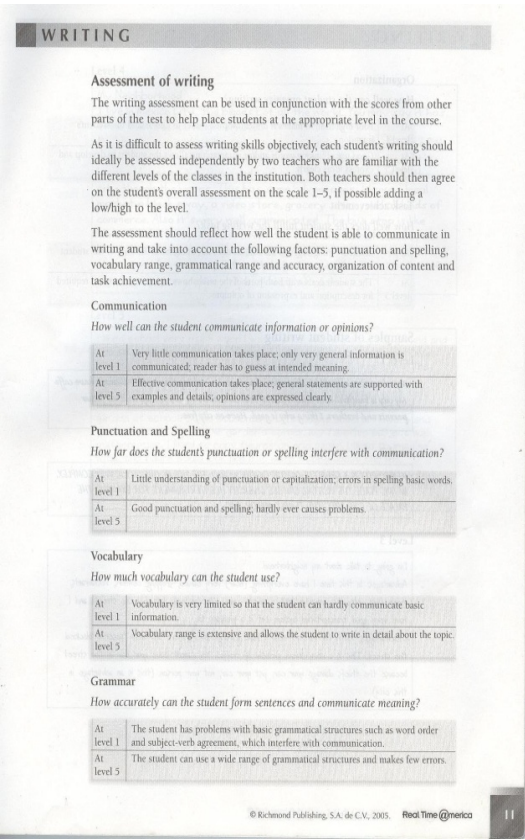

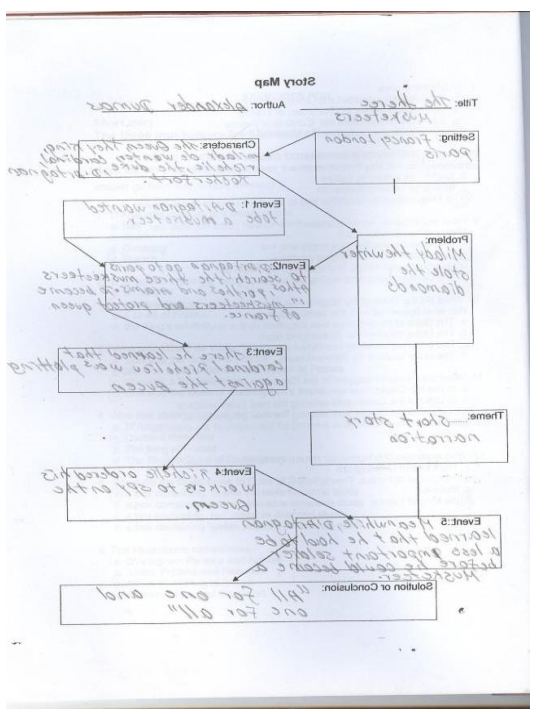

INIEES - UPNFM 
Incorporación de la literatura en un programa lectura del idioma inglés para

desarrollar la competencia de escritura: Instituto Blanca Adriana Ponce de Tegucigalpa

\section{Referencias Bibliográficas}

Axbey, S. (2005). Real Time @merica, Placement Test; Richmond Publishing; S.A. de C.V. Av. Universidad 767,03100 Mexico, D.F. 18 Berghem Mews; Blythe Road; London W14 OHN

Beltran, R. Ch, Senra I., Lawley J.; Fernández R. C. (2010). Everything 3A. Richmond Publishing 58 St. Aldates, Oxford, OX1 1st England. ISNB: 978607-6-00099-1

Burdett, S. Company (1984). The Three Musketeers by Alexander Dumas. Adapted for young readers by Vincent Buranelli

Burns, A. (2009). Action research: an evolving paradigm. Department of Linguistics, Macquarie University, Sydney Cambridge J ournals

Pie de hierro, C. (2009). La transferencia de instrucción. Un rasgo de la Inter lengua de aprendices francófonos de Helé revista Lebrija de Lingüística Aplicada a la Enseñanza de Lenguas. Ëcole des Hautes Ëtudes en Sciences

Castellanos, J . (2008). J ournal Writing and its Benefits in an Upper Intermediate EFL Class. La escritura por medio de diarios y sus Beneficios en una clase de nivel Intermedio

República de Honduras Secretaria De Educación Subsecretaria Técnico Pedagógico Dirección General De Currículo. "Currículo Nacional Básico" (2004). Tegucigalpa. M.D.C. Honduras, C.A. 2004. doc, pdf.

Smith, C.C. (1984). The Gull that Lost the Sea. Illustrated by Lucinda McQueen. A Golden Book, New York. Western Publishing Company, Inc. Racine, Wisconsin.

Clandfield, L. (2009). Teaching materials: using literature in the EFL/ESL classroom. http://www.onestopenglish.com/ section. asp?docid=146508» 
Clara Elizabeth Chávez Suazo

Finch, A.E. (1998). Designing and using a Learner J ournal for false Beginners: Self- assessment and Organization of Learning. Andong National University

Muñoz, S. J . (2003). El uso de Textos Literarios en la Enseñanza de Inglés En la Educación Secundaria. Departamento de Lengua Inglesa Facultad de Filología y Letras. Universidad de Granada, Granada España. 